\title{
Development and evaluation of a community respiratory physiotherapy service for children with severe neurodisability
}

\author{
Antonia Wolff, Hayley Griffin, Moira Flanigan, Sarah Everest, David Thomas, William Whitehouse \\ Nottingham University Hospitals NHS Trust
}

\begin{abstract}
Children with severe disability often have difficulties with secretion clearance leading to recurrent lower respiratory tract infections and prolonged hospital admissions. A community respiratory physiotherapy service was developed for this client group, including a rapid response for acute respiratory illness, and an evaluation was undertaken, comparing admissions and bed-days in hospital for respiratory tract infections, emergency admissions to hospital for any cause, and admission costs for each child for the 12 months before to the 12 months with the service.

Thirty-four children aged 1 to 19 years (median 5) were eligible for the "before and after" evaluation at 28 months; most had severe cerebral palsy $22(65 \%)$, and there were also eight (23\%) children with neurodegenerative and four (12\%) with neuromuscular conditions. Admissions for respiratory tract infection fell from 43 to $25(p<0.05)$, respiratory admission bed-days fell from 383 to $236(p<0.01)$, total non-elective admissions fell from 64 to 40 ( $p<0.01$ ), with admission cost savings of GBP $78,155(52 \%)$ per annum.
\end{abstract}

This small study suggests that a community respiratory physiotherapy service can reduce hospital admissions and bed days for disabled children. The admissions cost savings have enabled the service to "pay for itself".

\section{Problem}

The neurodisability team of Nottingham University Hospitals NHS trust provides medical and nursing support in hospital and community settings to the children of Nottingham City and South Nottinghamshire, UK; a population of 150,000 children aged 0 to 18 years. The team includes paediatric neurologists, neurodisability, and respiratory paediatricians, hospital based respiratory physiotherapists, and community nurses. The team was aware that many severely disabled children on their caseload were having frequent and prolonged hospital admissions for respiratory tract infections and once in hospital their discharge was often delayed by the need for chest physiotherapy to aid secretion clearance after an acute illness.

Many parents and carers stated in discussions regarding the emergency healthcare plans for their child that avoiding hospital admissions was a key priority.

\section{Background}

There are increasing numbers of children and young people with severe disability and complex medical needs living in the community aided by technologies such as gastrostomy, tracheostomy, and home ventilation.[1,2] They often have problems with coordination of swallow, gastro-oesophageal reflux, scoliosis, restrictive lung disease and respiratory secretion clearance which put them at risk of recurrent chest infections. [3] They therefore have frequent emergency department attendances and hospital admissions, which tend to be prolonged and may involve paediatric intensive care (PICU). [4,5] They consume considerable emergency and acute paediatric health services.[6]

It is widely agreed that children with disabilities should have early intervention from flexible services provided in a coordinated and timely manner, and that care should be provided closer to home, with hospitalisations minimised.[7,8,9] In addition, preventing lower respiratory tract infections in children from becoming serious is a recommended outcome in the NHS Outcomes Framework 2015-16.[10]

It has been shown that caregivers can be trained to carry out airway clearance techniques in a home environment safely and effectively for children with neuromuscular conditions. They can then be supported in their own environment during acute episodes of respiratory infection sometimes avoiding the need for hospital admission.[11,12] The use of regular airway clearance techniques is recommended for children with neuromuscular conditions by the British and American Thoracic Societies.[13,14] Chest physiotherapy is also widely used in the acute hospital management of chest infection in children with severe disability with non-neuromuscular conditions such as severe cerebral palsy although the evidence of its effectiveness is not established.[3,15]

A children's respiratory physiotherapy service, which includes a rapid response to children in the community when acutely unwell has been operating in one district of London, UK (Tower Hamlets) since 2009. That team has presented data indicating that hospital admissions can be reduced by such a service.[unpublished data]

\section{Baseline measurement}


An initial group of 20 children with severe neurodisability, and secretion clearance problems or recurrent chest infections were identified by the respiratory and neurodisability paediatricians. These 20 children had together had 50 emergency department attendances and 38 acute hospital admissions for respiratory tract infections in the 12 months prior to the onset of the community respiratory physiotherapy service.

See supplementary file: ds5320.doc - "example chest physio programme \& satisfaction questionnaire"

\section{Design}

Inspired by the service in Tower Hamlets, London, we aimed to develop and evaluate a children's community respiratory physiotherapy service for severely disabled children at risk of recurrent hospital admissions for respiratory tract infections, including home ventilated children and children with life limiting and life threatening conditions. The aim of the service was to assess and treat in the community, prevent emergency department attendances and hospital admissions, and reduce length of hospital stay. Thus this would improve the health, participation, and quality of life of these children and their families.

A project group involving clinicians and commissioners was established to plan, evaluate, and identify opportunities for ongoing funding for the service. We chose to use a retrospective "before and after" observational method, comparing outcomes within each child rather than try to have a control group because these patients are few in number and have a large variety of underlying conditions many of which will deteriorate over time.

The group of children we were planning to serve were fragile and had complex health needs, therefore a respiratory physiotherapist was seconded from the hospital team who was highly experienced in managing acutely ill children, trained to use the paediatric early warning score (PEWS) [16] and who continued to participate in the on call hospital rota.

An initial caseload of 20 children and young people with severe disability and recurrent chest infections was set, based on predicted capacity for a single therapist and the recognition that establishing the service and developing tools and pathways would be time consuming.

All the children were already known to the clinicians involved in the project as it was important that the prognosis and treatment objectives were discussed and agreed with the family and, where possible, the young people themselves, so that the intensity and invasiveness of the care was appropriate. For example, it needed to be agreed whether the use of deep suction or cough assist devices was appropriate, and that clearly written family held respiratory care, emergency healthcare, and personal resuscitation plans, $[17,18]$ needed to be developed for each child by their clinician. These included symptoms and signs indicating deterioration, and action to be taken, including when to call out the rapid response respiratory physiotherapist urgently.
Only children living within a travel time of 30 minutes (15 mile radius) of the base hospital were accepted to make the rapid response in acute deterioration feasible.

The intervention comprised the following:

1. A specialist assessment of the child's respiratory condition by the community respiratory physiotherapist in conjunction with the child's medical team

2. Development of a daily tailored chest physiotherapy programme, including manual techniques, suction airway management, and use of equipment to increase lung volumes and instigate cough

3. Training of the parents and carers, including professionals in school, short break (respite) facilities, and hospices, in the specific chest physiotherapy programme for each child

4. A rapid response respiratory physiotherapy service, for whenever the child became acutely unwell, in whatever setting, 8:30 a.m. to 4:30 p.m., Monday through Friday, including assessment using the paediatric early warning score (PEWS). The respiratory physiotherapist would provide emergency treatment using specialist equipment as required eg a lung volume recruitment (LVR) ambu bag, or cough assist device.[13] The physiotherapist would then review the child on a daily basis as needed, collect sputum and liaise with the paediatrician or family physician/general practitioner (GP) to source antibiotics when appropriate, and advise admission only when necessary

5. For children in hospital, the therapist would liaise with the ward and intensive care staff to facilitate early hospital discharge and continue the chest clearance techniques in the community.

We developed the following tools:

- Information letters for parents/carers and general practitioners

- Respiratory physiotherapy care pathway and flow diagram

- Individual respiratory assessment form

- Competency form for parents and professionals in each child's community care team

- A service satisfaction tool to be given to parents and carers after receiving the service for 12 months.

The main performance indicators were the number of emergency department attendances and the number and duration of hospital admissions for respiratory tract infections in the 12 months pre and post service onset for each individual child. An admission was to be identified as "respiratory" when respiratory tract infection was the primary diagnosis on the discharge summary.

Statistics: Each child would acted as their own control and Wilcoxon signed ranked matched pairs statistical test used to compare performance indicators before and after introduction of the service for each individual child. We also planned to record the number of 
'rapid response' visits and the number of treatment days in the community for each child, and also whether the child was receiving or was started on prophylactic antibiotics, home ventilation and / or enteral feeding. Information regarding deaths of children who had been referred to the service and of local children with neurodisability would be obtained from the district child death review database.[19]

See supplementary file: Service Satisfaction

\section{Strategy}

The service development group met at four to six monthly intervals with the respiratory physiotherapist to review cases, the key performance indicators, service activity, user feedback, and quality issues.

PDSA cycle 1: Review at four months indicated that admissions were being avoided for some children but not all families were using the service appropriately and not all the families were remembering to use the rapid response component of the service. The user information leaflet was revised and re issued. Several awareness raising presentations were made to staff in hospital and community settings and professionals were urged to remind families to access the service rather than go to the emergency department. The paediatricians reviewed the individual emergency healthcare plans with parents and carers at clinic appointments, particularly emphasising when to call out the therapist.

Many of the children had very large teams of carers in school, short break unit, hospice and home, and the training of all these carers needed to be coordinated for maximum efficiency. The therapist started to provide group training sessions for general respiratory assessment skills but continued individual patient specific training for chest clearance techniques. Feedback forms for community staff were developed.

We discovered that there needed to be additional funding for consumables i.e suction catheters, tubing, nasopharyngeal aspirate kits, gloves, alcohol gel.

PDSA cycle 2: At 12 months, data showed a reduction in emergency department attendances and hospital admissions for the initial group of 20 children and user satisfaction was extremely high. All the families reported improved confidence in managing their child's chest problems. Feedback from staff in community settings was very positive, both regarding their improved confidence in the management of individual children's respiratory needs but also regarding the timeliness and usefulness of the rapid response service. A further 12 children had been referred to the service, some of whom were acutely unwell at the time of referral. The referral process was standardised and a referral form was developed. Maximum "referral to treatment onset" time for urgent cases was agreed to be 48 hours and for non urgent cases, two weeks. Commissioners agreed to fund the service for a further 12 months.

One of the original 20 children had died following a period of end of life care and others were deteriorating due to their degenerative conditions. It was agreed that the respiratory physiotherapist would attend the multiagency reviews for these children to contribute to discussions regarding prognosis and care planning.

There were delays in accessing sputum results and antibiotics via the GP and therefore we established a new pathway for handling cough swab and sputum samples. The therapist collected the samples and sent them to the hospital microbiology laboratory with the respiratory paediatrician (DT) named on the request forms. The therapist could then access the patient electronic record for the results and arrange prescription of appropriate antibiotics via the consultant paediatrician or GP.

PDSA cycle 3: At 28 months, 68 children had been referred to the service and the physiotherapist was struggling to provide all the training and respond to the emergency calls in a timely manner. Feedback from families remained very positive, however several had commented that they wanted a service at week ends. Admissions and emergency department attendances had been reduced but some children were being admitted to hospital when the therapist was unavailable ie week ends and annual leave.

The community and respiratory paediatricians estimated that 80 to 100 children and young people across Nottinghamshire could benefit from the service. A business case was written with estimated cost savings based on the average admission tariff, however actual cost savings data were required. Therefore the numbers and costs of all non-elective hospital admissions for each child in the 12 months pre and post service onset was obtained from the secondary users service (SUS) data warehouse which provides data for audit, and healthcare planning.[20] Actual cost savings were demonstrated and the local health commissioners then agreed to fund a permanent service with two physiotherapists to increase the service capacity to 80 children and extend the service hours to include Saturdays.

\section{Results}

At 12 months, one of the original 20 children (a child with a neurodegenerative condition) had died. For the remaining 19 children, hospital admissions had reduced from 36 in the 12 months pre service onset to 24 in the 12 months with the service, and emergency department attendances had reduced from 48 to 33 . User satisfaction was very high and all families reported improved confidence in managing their child's chest problems.

At 28 months, 68 children had been referred to the service. Thirtyfour of 68 had been receiving the service for longer than 12 months and had their disabling condition for more than 12 months prior to service onset. Age at referral ranged from 1 to 19 years (median 5 years) and 21/34 (62\%) were female (see supplementary file: figure 1).

These 34 children had a wide range of disabling conditions. Twentytwo of $34(65 \%)$ had a non-progressive disability, most commonly severe cerebral palsy at level 5 in the gross motor function classification system (GMFCS),[21] 8/34 (23\%) had a 
neurodegenerative condition, and 4/34 (12\%) had a neuromuscular disorder. Five of $34(15 \%)$ were using long term home ventilation, one invasive; four non-invasive. In all cases this had commenced prior to the onset of the respiratory physiotherapy service. Sixteen of $34(47 \%)$ received regular prophylactic antibiotics (14 azithromycin, $10 \mathrm{mg} / \mathrm{kg}$ once daily on three days per week, and two trimethoprim, $2 \mathrm{mg} / \mathrm{kg}$ daily), including 11/34 (32\%) who had been started on prophylactic antibiotics before referral to the respiratory physiotherapy service. Twenty-three of $34(68 \%)$ children were entirely tube fed before commencing the service and 6/34 (18\%) were partially tube fed. One child with neurodegenerative disease changed from oral to tube feeding shortly after starting to receive the respiratory physiotherapy service, otherwise the mode of feeding of each child was unchanged.

The 34 children each had one to 31 (median 5) emergency call out episodes of care, comprising one to 80 (median 7) days of emergency treatment in the community by the respiratory physiotherapist in the first 12 months of receiving the service. $80 \%$ of the call out episodes consisted of a visit on one or two consecutive days. Only $4 \%$ of treatment episodes were longer than six days and none longer than 15 days (see supplementary file: figure 2).

These 34 children together had 43 acute hospital admissions for respiratory tract infections in the 12 months prior to each starting to receive the community respiratory physiotherapy service. This fell to 25 acute hospital admissions for respiratory tract infections in the 12 months after service onset. Paired data for 12 months pre and post service onset for each individual child showed a significant fall in acute hospital admissions for respiratory tract infection after service onset, $p<0.05$ (see supplementary file: figure 3 ).

The total number of bed days for respiratory tract infection fell from 383 in the 12 months pre service onset to 236 in the 12 months post service onset. Paired data for 12 months pre and post service onset for each individual child showed a significant reduction in bed days after service onset, $p<0.01$ (see supplementary file: figure 4).

The total number of attendances at the emergency department without subsequent hospital admission, for this group of 34 children, fell from 17 in the 12 months pre service to five in the 12 months post service onset. Number and cost of all non-elective admissions in this group of 34 also fell significantly $p<0.01$ ), with an admissions cost saving of $£ 78,155$ (52\%) per annum and a total cost saving per annum after subtraction of the cost of the service of $£ 19,624$ (see supplementary file: tables 1 and 2).

There were no adverse events attributable to the respiratory physiotherapy service. The number of deaths of children with neurodisability in this district was eight a year in the two years before the start of the programme and eight a year in the two years after it began. Five children had died within 12 months of referral to the service and had been excluded from the analysis. Each of these children had life shortening or life threatening conditions and the physiotherapy service was either being provided as part of the child's planned end of life care or had been withdrawn according to their end of life care plan.
Continuous parent / carer surveys of satisfaction showed $28 \%$ satisfied and $72 \%$ very satisfied on a five point scale.

See supplementary file: ds5773.docx - "fig 1-4, tables 1\&2, feedback from families"

\section{Lessons and limitations}

Although only a small study, this retrospective service evaluation indicates that a community respiratory physiotherapy service with rapid response to acute deterioration can reduce emergency department attendances, hospital admissions, and hospital bed days for children with severe disability and secretion clearance problems.

Previous studies have demonstrated the value of respiratory physiotherapy for children with neuromuscular conditions.[11,12] However, the majority of our cases had severe bilateral cerebral palsy (The gross motor function classification system (GMFCS) 5) and several children had a neurodegenerative condition where an increased number of admissions over time would have been expected rather than the decrease that was found. There was no increase in the district mortality rate for this client group and there have been no deaths attributable to the service.

From this study we are unable to identify which interventions from the new service are most effective. Each child received a tailored personal treatment plan both as a daily preventative regime and in an acute deterioration, devised and delivered by a specialist respiratory physiotherapist. We cannot say whether it was the daily preventative chest clearance programme or the rapid response in an acute deterioration which gave most benefit. All the children had received at least one rapid response call out visit. The number of respiratory physiotherapy treatment days in the community for an individual child did not correlate with the number of their previous hospital treatment days.

For some children, the rapid response physiotherapy treatment given in the community was very little, suggesting that it was the preventative daily programme that had the most positive impact; while some children had many community treatment days but no admissions before or after the service commenced. Several of the latter had neuromuscular and neurodegenerative conditions and would have been expected to have had more respiratory illness over time, which suggests that the service was beneficial. Feedback from parents and carers showed that they greatly valued the rapid response call out service and that they perceived that it had prevented hospital admissions for their child.

These children were receiving multiple medications and therapies. We were unable to analyse all the treatment changes that occurred during the period evaluated, however there were no significant changes in the use of home ventilation or prophylactic antibiotics. We did not assess the nutritional status of the children, which is known to be an important factor affecting a child's respiratory health,[22] however the mode of feeding changed for only one child during the study period: from oral to gastrostomy tube feeding. 
The new physiotherapy service did not operate in isolation. It was added to the support already provided by a local multidisciplinary team, which included paediatric neurologists, respiratory and community neurodisability paediatricians, community nurses and short break services.

There is a "hidden cost" of this service in the increased burden for the families and other care givers in keeping a severely disabled child at home when they become acutely unwell. Although feedback from parents and carers was very positive, families may become exhausted unless there is a flexible home care support package in place.[23]

An additional benefit from the service is that the respiratory physiotherapist provided specific training to all the professionals in each child's care team including staff in school, short breaks units, home care providers, and hospice staff. This training is likely to increase the skills in the community workforce, resulting in better support locally for all children with disability and respiratory symptoms.

It may be possible to study the benefits and burdens of the different elements of the service with larger numbers of children and treatment episodes, and also the different treatments used by the therapist (such as deep suction and cough assist technologies). The physiotherapist has subsequently become an independent prescriber and is able to prescribe appropriate antibiotics promptly according to sputum results, but continues to liaise closely with the child's medical team regarding medication decisions.

\section{Conclusion}

This evaluation suggests that a community respiratory physiotherapy service can lead to reduced hospital admissions and reduced hospital bed days for children with severe disability and can pay for itself in reduced admission costs.

We recommend that similar services should be developed for all severely disabled children at risk of respiratory tract infections. A suggested model would be to have several district teams linked to a regional paediatric respiratory care centre.

\section{References}

1. Cohen E, Kuo DZ, Agrawal R, Berry JG, Bhagat SK, Simon TD, Srivastava R. Children with medical complexity: an emerging population for clinical and research initiatives. Pediatrics 2011;127(3):529-38.

2. Fraser LK, Miller M, Hain R, Norman $P$, Aldridge J, McKenney PA, Parslow RC. Rising national prevalence of Life-Limiting Conditions in children in England. Pediatrics 2012;129(4):e923-29.

3. Seddon PC, Khan Y. Respiratory problems in children with neurological impairment. Arch Dis Child 2003;88:75-8.

4. Mahon M, Kibirige MS. Patterns of admissions for children with special needs to the paediatric assessment unit. Arch Dis Child 2004;89:165-69.
5. O'Mahony L, O'Mahony DS, Simon TD, Neff J, Klein EJ, Quan L. Medical complexity and pediatric emergency department and inpatient utilisation. Paediatrics 2013;131(2):e559-65.

6. Newacheck P, Inkelas M, Kim S. Health services use and health care expenditures for children with disabilities. Pediatrics 2004;114(1):79-85.

7. Shribman S. Making it better: for children and young people. London: Department of Health; 2007. Available at http://dera.ioe.ac.uk/6610/1/idcplg\%3FldcService\%3DGET FILE\%26dID\%3D122745\%26Rendition\%3DWeb (accessed 4 May 2015).

8. HM Treasury, Department for Education and Skills. Aiming high for disabled children. London: HM Treasury; 2007.

Available at

http://webarchive.nationalarchives.gov.uk/20130401151715/ http://www.education.gov.uk/publications/eOrderingDownloa d/PU213.pdf (accessed 4 May 2015).

9. Royal College of Paediatrics and Child Health (RCPCH) Submission to House of Commons Health Committee call for evidence on emergency services and emergency care. May 2013. Available at http://www.rcpch.ac.uk/news/rcpch-r esponse-health-committees-report-urgent-and-emergencyservices (accessed 15 July 2014).

10. NHS Outcomes Framework 2015-2016. Available at https://www.gov.uk/government/uploads/system/uploads/att achment_data/file/417894/At_a_glance_acc.pdf (accessed 4 May 2015).

11. Anderson JL, Hasney KM, Beaumont NE. Systematic review of techniques to enhance peak cough flow and maintain vital capacity in neuromuscular disease: the case for mechanical insufflation-exsufflation. Physical Therapy Reviews 2005;10(1):25-33.

12. Tzeng AC, Bach JR. Prevention of pulmonary morbidity for patients with neuromuscular disease. Chest 2000;118(5):1390-6.

13. British Thoracic Society Quality Standards for the Respiratory Management of Children with Neuromuscular Weakness. Available at https://www.brit-thoracic.org.uk/doc ument-library/clinical-information/children-with-nmw/quality-s tandards-for-the-respiratory-management-of-children-withnmw/ (accessed 4 May 2015)

14. Respiratory Care of the Patient with Duchenne Muscular Dystrophy. ATS Consensus Statement. March 2004. Available at https://www.thoracic.org/statements/resources/pldd/duchen ne1-10.pdf (accessed 4 May 2015).

15. McCrea N, O'Donnell R, Brown R. Outpatient respiratory management of the child with severe neurological impairment. Arch Dis Child Educ Pract Ed. 2013;98(3):84-91.

16. Paediatric Early Warning Score. Available at http://www.institute.nhs.uk/safer_care/paediatric_safer_care pews.html (accessed 4 May 2015)

17. Wolff A, Browne J, Whitehouse WP. Personal resuscitation plans and end of life planning for children with disability and life-limiting/life threatening conditions. Arch Dis Child Educ Pract Ed 2011;96:42-8. 


\section{BMJ Quality Improvement Reports}

18. Emergency Healthcare Plans for Children. Available at http://www.councilfordisabledchildren.org.uk/ehp (accessed 4 May 2015).

19. Guidance on child death review process. Available at http://www.workingtogetheronline.co.uk/chapters/chapter_fiv e.html (accessed 4 May 2015).

20. SUS data warehouse. Available at http://www.connectingforhealth.nhs.uk/systemsandservices/ sus (accessed 4 May 2015).

21. Palisano R, Rosenbaum $P$, Bartlett $D$, Livingston M. Gross Motor Function Classification System - E \& R, 2007.

Available at http://motorgrowth.canchild.ca/en/GMFCS/resources/GMFC S-ER.pdf (accessed 4 May 2015).

22. Fung EB, Samson-Fang L, Stallings VA, Conaway M, Liptak G, Henderson RC, Worley G, O'Donnell M, Calvert R, Rosenbaum P, Chumlea W, Stevenson RD. Feeding dysfunction is associated with poor growth and health status in children with cerebral palsy. J Am Diet Assoc 2002;102(3):361-73.

23. Leonard BJ, Brust JD, Nelson RP. Parental distress: caring for medically fragile children at home. J Pediatr Nurs 1993;8(1):22-30.

\section{Declaration of interests}

Nothing to declare.

\section{Acknowledgements}

The initial six months funding was from the UK Government "30 million stars" programme. The remainder was funded, as service development, by local National Health Service commissioners.

We are grateful to the Tower Hamlets Children's Community Respiratory Physiotherapy Service for sharing their experiences.

We are grateful to Deborah Hooton, head of joint commissioning for children and families, Nottingham City, and Pauline Pilling children's physiotherapy manager for their strenuous support to develop the service.

\section{Ethical approval}

This was a service evaluation conducted by clinicians who collected data from their own patients before and after the introduction of a new service. The chair of the local ethics committee agreed that this was a service evaluation and that it did not require formal ethics committee approval. 